\title{
Virtual Knowledge Production within a Physician Educational Outreach Program
}

\author{
Mowafa Said Househ* \\ College of Public Health and Health Informatics \\ King Saud Bin Abdul Aziz University for Health Sciences \\ National Guard Health Affairs \\ Kingdom of Saudi Arabia, Riyadh \\ E-mail: househmo@ngha.med.sa
}

\author{
Andre W. Kushniruk \\ School of Health Information Science \\ University of Victoria, Victoria, BC, Canada \\ E-mail: andrek@uvic.ca
}

\section{Malcolm Maclure}

School of Health Information Science

University of Victoria, Canada

Pharmaceutical Services Division

British Columbia Ministry of Health, Victoria BC, Canada

E-mail: mmaclure@uvic.ca

\section{Bruce Carleton}

Pediatrics and Pharmaceutical Sciences

University of British Columbia, Canada

Children's and Women's Health Centre of BC

Child \& Family Research Institute, Canada

E-mail: bcarleton@popi.ubc.ca

\section{Denise Cloutier-Fisher}

Department of Geography

University of Victoria, Victoria, BC, Canada

E-mail: dcfisher@uvic.ca

*Corresponding author

\begin{abstract}
This paper describe the impacts and lessons learned of using conferencing technologies to support knowledge production activities within an academic detailing group. A three year case study was conducted in which 20 Canadian health professionals collaborated on developing educational outreach materials for family physicians. The groups communicated in face-to-face, teleconferencing, and web-conferencing environments. Data was collected over
\end{abstract}




\begin{abstract}
three years (2004-2007) and consisted of structured interviews, meeting transcripts, and observation notes. The analysis consisted of detailed reviews and comparisons of the data from the various sources. The results revealed several key findings on the on the impacts of conferencing technologies on knowledge production activities of academic detailers. The study found that: 1) The rigid communication structures of web-conferencing forced group members to introduce other tools for communication 2) Group discussions were perceived to be more conducive in face-to-face meetings and least conducive teleconferencing meetings; 3) Web-conferencing had an impact on information sharing; 4) Web-conferencing forces group interaction "within the text". The study demonstrates the impacts and lessons learned of academic detailing groups collaborating at a distance to produce physician education materials. The results can be used as the bases for future research and as a practical guide for collaborative academic detailing groups working within a virtual collaborative and educational environment.
\end{abstract}

Keywords: Conferencing technologies; Web-conferencing; Distance education

Biographical notes: Mowafa Said Househ is an Assistant Professor, College of Public Health and Health Informatics, King Saud Bin Abdul Aziz University for Health Sciences, National Guard Health Affairs, Kingdom of Saudi Arabia, Riyadh. He received his $\mathrm{PhD}$ in Health Information Science from the University of Victoria in Canada.

Andre W. Kushniruk is a Professor in Health Information Science at the University of Victoria. He has expertise in the area of health informatics, human factors and development of educational programs in health informatics. He holds a PhD from McGill University.

Malcolm Maclure DSc is an Adjunct Professor at the School of Health Information Science, University of Victoria, Canada. He also works for the Pharmaceutical Services Division, British Columbia Ministry of Health, Victoria BC, Canada.

Bruce Carleton, PharmD, is Professor of Pediatrics and Pharmaceutical Sciences, University of British Columbia. He is Director, Pharmaceutical Outcomes and Policy Innovations Program, and is affiliated with the Children's and Women's Health Centre of BC, and is Senior Clinician Scientist, Child \& Family Research Institute.

Denise Cloutier-Fisher, $\mathrm{PhD}$, is Associate Professor in the Department of Geography at the University of Victoria. She is Scientific Director, (UVic Site) for the BC Rural and Remote Health Research Network, sponsored by the Michael Smith Foundation for Rural and Remote Health Research.

\title{
1. Introduction
}

Within the healthcare field, a growing interest in the use of information and communication technologies (ICT) has encouraged decision-makers, researchers, and clinicians to collaborate on the production, dissemination, and use of research knowledge to support clinical and administrative decision-making. The purpose of these research partnerships is to produce research knowledge that can improve health, produce more effective services and products, and ultimately, strengthen the health care system (CIHR, 
2002). Academic detailing is a form of educational outreach that is based on the creation of such partnerships between clinical, research, and administrative groups to improve clinical decision-making and health and cost-effectiveness of care (Soumerai and Avorn, 1990). Academic detailing is a process that involves studying the status of how knowledge is used; defining knowledge and its objectives; including practitioners and opinion leaders; and providing support for the implementation of research knowledge (KUSP, 2003). Academic detailing methods have been used by non-profit organizations to reduce inappropriate prescribing by clinicians (Soumerai and Avorn, 1990). However, much of the research on academic detailing has focused on the process of disseminating educational material to clinicians, with little focus on the production of knowledge within academic detailing groups (O'Brien et al., 2007). Furthermore, little research is known about the impacts of virtual collaboration on group work and knowledge production within an academic detailing environment.

Accordingly, this paper details findings relating to the impacts of virtual collaboration on knowledge production processes within an academic detailing group. The paper aims to describe the experiences and lessons learned of an academic detailing group working within a virtual environment on academic detailing activities.

\section{Methods}

The case study method was used to study the knowledge production process of academic detailing groups working within a virtual environment. The research study took place between 2004 and 2007. Case study research appears in the social sciences, health informatics, knowledge translation, and the ICT literature. A widely accepted research methodology, it serves to describe the real-life context in which an intervention has occurred and demonstrates the details of participant viewpoints through the use of multiple data sources (Stake, 1995; Yin, 2003). Yin (2003) and Stake (1995) note that case study is a method that is all-encompassing and includes design, data collection, and analysis strategies. They are a preferred strategy if an investigator is interested in knowing how something happened and why it may have happened (O'Sullivan \& Rassel, 1999).

\section{Participants}

The participant group consisted of academic detailers who produced research reviews regarding new drugs for dissemination to physicians. Of the 26 potential participants in the group, 20 were included in the study. The six that were excluded were observers and administrative assistants. The 20 participants included in the group were researchers, educators, and decision-makers. The group was comprised of ten $(50 \%)$ men and ten $(50 \%)$ women. Of the 20 , three $(15 \%)$ were decision-makers; three (15\%) were researchers; and $14(70 \%)$ were educators. All participants had at least a bachelor's degree. Thirteen of the educators were pharmacists by training and one was a physician. All three researchers were experienced drug policy researchers. The educators were from five Canadian academic detailing programs located in British Columbia, Alberta, Saskatchewan, Manitoba, and Nova Scotia. A one- to ten-year range of experience in academic detailing was noted for the different academic detailing programs. All the researchers involved in the education task group were based in British Columbia, and each had over ten years of drug policy research experience. The list of participants was obtained from an administrative member of the education task group. Consent to participate was obtained via e-mail. Consent was also obtained from participants during a 
teleconference call held in March 2004. No incentives to participate in the study were offered or given to the educators, researchers, or decision-makers. All group members had experience using e-mail, teleconferencing, and desktop computers.

\section{Technologies Used}

The web-conferencing technology used in this study was Elluminate Live V-Class edition. This version allowed for half-duplex audio communication that permitted users to speak one at a time. Full-duplex technologies allow more than one participant to speak at a time, which is currently a standard feature of Elluminate and other major web-conferencing providers, such as, Web-Ex. Elluminate allows users to upload agendas to the whiteboard, share documents via application sharing, use instant text messaging, vote/poll participants, use emoticons, raise hands, and see participant names. These were the most relevant features used by the groups in this study. For example, power point slides were uploaded to the white board. Voting/Polling and Emotion indicators were used in performing communication checks and demonstrating satisfaction with a comment or point a group member made. The hand raising feature and chat box were used when someone wanted to make a point or speak to the group.

Figure 1 shows a generic screen shot of the Elluminate Live technology. The most visible feature is the participant window (top left). In this window, the participants' names are displayed along with emoticons and the hand raising features. Below this window is the direct chat messaging feature, which allows group members to communicate using text messages. Below the direct messaging window is the half-duplex audio communication function. The group used this function to communicate using audio. The main screen is the whiteboard page. This allowed group members to share and manipulate PowerPoint slides using the whiteboard tools.

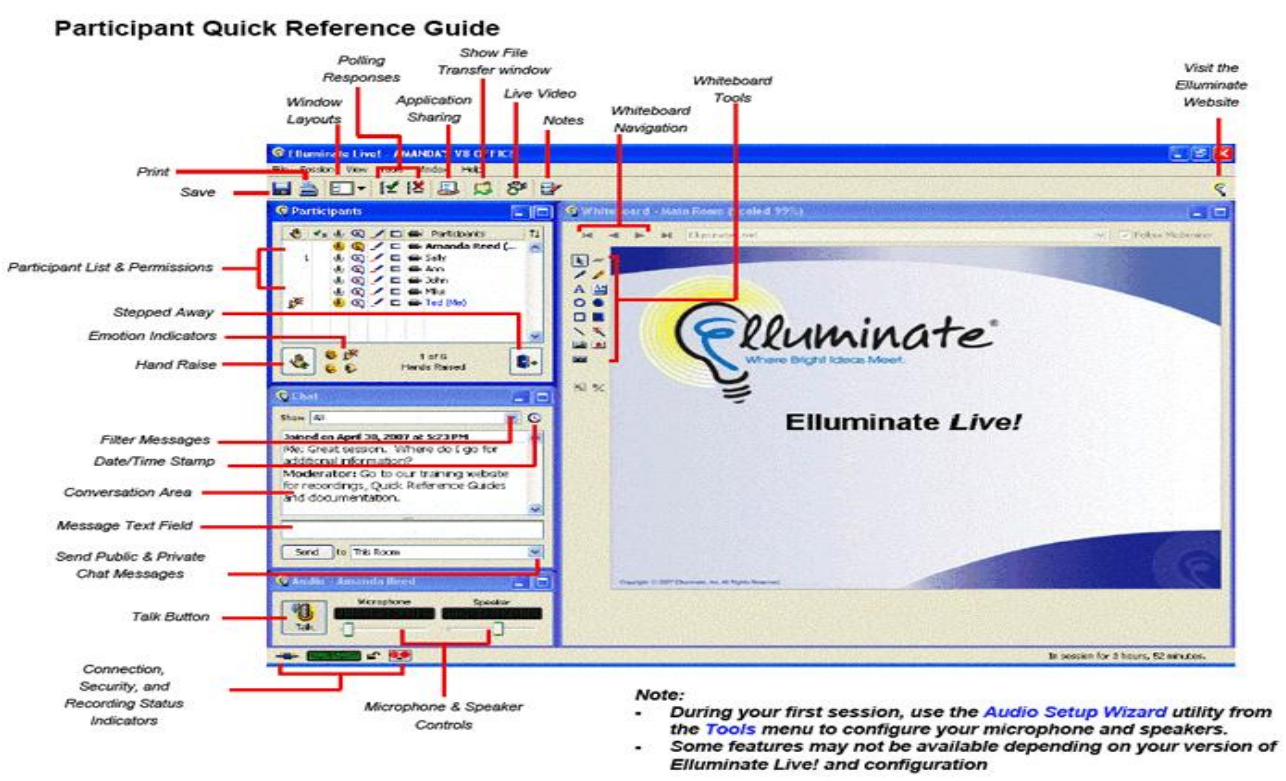

Figure 1. Elluminate Live Screen Shot. 
For teleconferencing, the group used audio teleconferencing technology. The technology used audio-only communication in which multiple participants could speak at a time and there was no video or other media for communication. A participant simply dialled a telephone number, entered a conference code, and responded to a prompt requesting his or her name. A beep let other participants know that someone had joined the meeting. To use this technology, group members needed access to e-mail and a telephone. E-mail was necessary to inform the participants about the meeting details (i.e., time, numbers to dial, and the agenda). A telephone was required to participate in the meeting.

\section{Group Tasks and Meeting Structure}

The group communicated using face-to-face, teleconferencing, and web-conferencing from January 2004 to December 2006. The formation of the group began in 2004 with a purpose to collaborate on new initiatives, learn from each other, and develop academic detailing material. Since it was a large group, dispersed in several Canadian provinces, weekly or monthly face-to-face meetings were not possible. The group decided to use teleconferencing and gradually move towards the use of web-conferencing technologies to support group work.

From September 2004 until December of 2004, the group met weekly using teleconferencing technologies. The teleconferencing meeting occurred every third Tuesday of every month with a rotating chair whose role was to facilitate the group process. Prior to the teleconferencing meetings, the meeting chair would, via e-mail, send meeting information (e.g., time, conference number and agenda) to the group. The meeting chair would also send any relevant documents that were relevant to the meeting. On the day of the meeting, group members logged in a few minutes prior to the meeting and the chair would begin the meeting sticking to the agenda items. The meetings would last between 60-120 minutes. Sample topics discussed in the education task group teleconferencing meetings included communication needs, physician education materials, relationships with funding agencies, a literature synthesis project, roles and relationships, and group identity issues.

In January of 2005, the group started to meet via web-conferencing, which is an internet based tool with several features (e.g., video, slide sharing, recording, text chatting) to support group communication. Similarly, to the teleconferencing meeting process, the group would meet every third Tuesday of every month with a rotating chair. Agenda items were sent in advance along with a link to the web-conferencing meeting. Group members were asked to $\log$ in at least 20 minutes earlier than the designated meeting time so that any technical glitches could be addressed. The chair placed agenda items on the whiteboard and performed a sound check. During the sound check, the facilitator asked group members to speak into the microphone. If they heard the speaker clearly, they would signal using the voting and polling feature with the checkmark. If they did not hear the speaker clearly, they would signal with an $\mathrm{x}$. The meeting lasted between 50 minutes and 120 minutes. The group followed the agenda and crossed out items as they finished them. Sample agenda topics included grant proposals, physician education materials, literature, time lines and deliverables for the group evaluation project, and status reports.

\section{Data Collection}

Baseline interviews and observations, meeting transcript data, and semi-structured interviews were collected over a three year period (2004-2007). Baseline questions 
consisted of probes asking the group participants about group behaviour, communication modes, and technology perceptions. Baseline interviews for the group were conducted between June and August 2004 with 12 group members interviewed. Of the 12 respondents, two were researchers, nine were educators, and one was a decision-maker. Eight of the 20 participants were not available to interview for the following reasons: they referred to another subject within the group to speak on their behalf; it proved difficult to schedule an interview with them; or they participated at a later stage of the study.

Throughout the study, between January 2004 and November 2006, the researcher collected electronic notes, which were stored on a computer, in order to acquire observation data. Using Microsoft Word and Excel, the researcher recorded notes informally every two to three months. The notes primarily concerned the evolution of the education task group and its relationship with other groups.

Web-conferencing meetings were recorded with a computer monitor using Windows Media Encoder, which included the audio and web components of the meeting. Similarly, teleconferencing sessions were recorded using a digital voice recorder with a telephone attachment to assure a higher quality recording. Because of time and budget constraints, the study included only four out of six teleconferencing meetings: March, May, July, and August 2004. Again, because of constraints, of the ten web-conferencing sessions, only four were included: April, June, August, and November 2005. One face-toface session, June 2004, was recorded.

Post-interviews were conducted with key informants in the education task group. Of the seven respondents interviewed, five were educators, one was a researcher, and one was a decision-maker. The interviews were conducted between May and July of 2007 and were all taped and transcribed. The post-interviews were recorded using a digital voice recorder with an attachment to ensure the quality of the recording.

\section{Data Analysis}

Content analysis was utilized to assess observational data, meeting transcripts and postinterview data. In general, content analysis is a data analysis approach that can be used to analyze qualitative data; it is a systematic process of analyzing communication messages and making inferences based on the analysis (Berg, 1989; Kondracki, Wellman, \& Amundson, 2002). Content analysis involves the interpretation of textual data that has been categorized into concepts. Once the identification of concepts or categories has taken place, they are categorized into themes based on their relationships with each other (Lau \& Hayward, 2000). For the analysis, groups of phrases or sentences provided an appropriate level of sampling for the post-interview and meeting transcript data. A subsequent step in the analysis was to extract and compile all of the responses. In this process, responses from the group were separated from each other. The researcher familiarized himself with the data by reading the responses to post-interviews and meeting transcripts for each of these groups. With meeting transcripts and post-interviews, sentences and phrases were selected as the basic units of analysis. As for survey data, summary statistics such as frequencies and averages were used. 


\section{Results}

Several findings relating to virtual collaboration and knowledge production within the academic detailing program did emerge. Table 1 provides a summary of the lessons learned.

Table 1. Four key lessons learned on the impacts of conferencing technologies on Academic Detailing Groups

\section{Lessons Learned}

Finding 1: The rigid communication structures of web-conferencing forced group members to introduce other tools for communication.

Finding 2: Group discussions were perceived to be more conducive in face-to-face meetings and least conducive in teleconferencing meetings

Finding 3: Web-conferencing had an impact on information sharing.

Finding 4: Web-conferencing forces group interaction "within text".

\section{Finding 1: The rigid communication structures of web-conferencing forced group members to introduce other tools for communication}

Because web-conferencing technology allowed one person to speak at one time, respondents noted that this limitation restricted group discussions around the production of academic detailing material. For example, the technology made it difficult to interrupt the speaker. Because of these limitations on voice communication in web-conferencing meetings, the group used text messaging in the meetings for asking questions, troubleshooting, and communicating generally; as well, group members used emoticons to express laughter and applause. This demonstrated that when encountering a highly structured approach to communication, the group members resorted to other forms of communication to compensate for the lack of socio-emotional interactions found in webconferencing. The two statements below support the above finding:

Just in the sense that if somebody was talking on and on and you wanted to interrupt them you wouldn't be able to do it vocally. You would have to do it via e-mail or a little text note or something like that [i.e., emoticons].

I recall one of my problems is that I tend to interrupt when I get excited about a point. That was less possible with web-conferencing because you had to take turns more.

An explanation for this phenomenon may be that as the group moved from unstructured communication environments (i.e., face-to-face or teleconferencing) to the highly structured web-conferencing, the dynamics of group communication changed. With web-conferencing, group members could only communicate vocally one person at a time using the hand raising function. The groups compensated for this loss of immediacy in communication by adapting and introducing new methods and structures for communication. Group members began using text messaging and emoticons as a way to improve group socio-emotional interactions. 
This finding agrees with predictions of the theory of adaptive structuration (AST) (DeSanctis \& Poole, 1994). According to AST, the use of ICTs introduces structures that influence the rules and resources that govern social interactions. Within both the education and research task groups, new forms of interaction norms were introduced to moderate the influence of technology on group social interactions.

\section{Finding 2: Group discussions were perceived to be more conducive in face-to- face meetings and least conducive in teleconferencing meetings}

The academic detailing group noted that face-to-face meetings were perceived as the most conducive structure for producing academic detailing material. Discussions were richer due to non-verbal communication, more spontaneous due to free flowing discussions, and more immediate with regard to responses to group discussion. During teleconferencing meetings, discussions were perceived to be not as rich because no nonverbal cues could be shared. However, the discussions in teleconferencing meetings were found to be similar in immediacy and spontaneity to face-to-face meetings. Webconferencing was perceived to be a richer form of communication than teleconferencing because group members could use emoticons, voting and polling, whiteboard, and text messaging. However, the groups found that web-conferencing reduced spontaneity and immediacy of discussions. Several members noted their preference for web-conferencing because it allowed them to share articles with the group and power point slides. This was found to help in producing academic detailing material.

\section{Finding 3: Web-conferencing had an impact on information sharing}

Respondents noted that technology had an impact on how information was shared in the group and not the level of evidence. For example, web-conferencing helped ensure that group members were looking at the same information material displayed on the whiteboard. However, group members felt that the web-conferencing forced them to summarize information in order to share it with group members. The two statements below demonstrate this:

In a face-to-face meeting they have materials and articles in front of them, and it is easier for them to reference the articles because they can see what others are referencing. As a chair, you don't know if they are looking at the right one or not. You can ask them, but you are still not sure if they are or not. In web-conferencing at least you can ensure they are looking at the same material.

With an academic detailer who has detailed information, they would feel web-conferencing as an impediment to sharing detailed information with other group members. In the future, I can imagine either larger screens or pairs of screens where they are looking at the entire document. Then technology would be better if you could see a full page document plus all of the web-conferencing features.

Though respondents noted that technology did not affect the strength of the evidence, they acknowledged that technology did affect how the evidence was shared within the group, as the statement below demonstrates this:

We're all pretty much evidence based people, and I don't think anybody would explicitly disagree that web-conferencing affected the level of evidence. 
In general, the ICT literature on information exchange has focused on types of information (i.e., task, social, and contextual information), distribution of information, information sharing challenges, and general effects of technology on information sharing (Cramton \& Orvis, 2003). Only a nominal amount of literature discusses the effects of technology on the level of evidence and how information is shared.

\section{Finding 4: Web-conferencing forces group interaction "within the text"}

Group members noted that when group members started to use web-conferencing to produce academic detailing material, their interactions changed from interacting with each other to interacting within the text. For example, when the groups met face-to-face, group members would face each other directly and share the same space. In webconferencing, however, the agenda would be placed on the whiteboard for all to see. All members would interact with and talk about the agenda placed on the whiteboard. The whiteboard itself represented the shared space, whereas in face-to-face meetings the shared space was the office or room. Group interactions within web-conferencing were structured around the text, and the documents were shared on the whiteboard, as demonstrated by the following statement:

[Web-conferencing] allows people to engage visually in addition to being able to talk and listen, which is a key component particularly when a lot of our work was going through education materials. So [in web-conferencing], for example, everybody is looking at the same thing on a screen, and that kind of keeps the group together for one, and it also helps make sure that we're all talking about the same thing.

Cramton $(2002,2001)$ conducted studies on the effects of text-based interactions on group communication. In studies of a performance appraisal system design firm, the author observed that due to the absence of non-verbal cues in e-mail messages, group members did not pay attention to certain messages embedded within the e-mail. The author noted that text-based interactions, especially e-mail communication, can lead to group members missing text-embedded messages. Though Cramton's (2002, 2001) studies focused on e-mail messages, it is important to note the potential ramifications of text-based interactions with regard to how information is processed by group members. The potential implication for knowledge exchange is that pieces of information may be missed or misinterpreted by group members.

\section{Discussion}

This study provided new insights into the impacts of conferencing technologies on the knowledge production process of academic detailers. The findings of the study demonstrate that using conferencing technologies had several advantages and disadvantages to the academic detailing group. The academic detailing group found webconferencing enforced a rigid communication structure within the group that led them to use other forms of communication to collaborate on producing academic detailing material. The group also preferred communicating in face-to-face meetings, however, that was not feasible given the size and geographical dispersion of the group. The group used teleconferencing and then gradually moved to using web-conferencing technology. The group preferred the use of web-conferencing because it was richer in communication than teleconferencing: web-conferencing provided a whiteboard, chatting, use of emoticons, voting/polling, and other features that enhanced communication. The group also noted that using the web-conferencing limited the amount of information they could 
share due to the size of a computer screen. Last, because of sharing text via the computer screen, group members felt that they were interacting through the text via the webconferencing whiteboard.

The study demonstrates that the use of conferencing technologies can help groups communicate and collaborate at a distance on the production of research knowledge. Within the healthcare education literature, little research is available on evaluating conferencing technology on health education (Valaitis, et al., 2007). Similar to the conclusions found in this study, Valaitis et al. report that conferencing technologies can be viewed as an enabler especially when face-to-face meetings are not possible (2007). Quintana et al. studied online internet learning networks that deliver various educational programs to nurses and doctors around pediatric cancer and aids using web-conferncing technologies. They used similar tools used in this study such as the whiteboard, power point slides and half-duplex technology. The study found that conferencing technologies allowed them to expand their educational programs regionally and internationally. The authors note that without the use of web-conferencing, the level of participation may have not been as high (2003).

Also based on social presence theory, which suggests that a higher degree of interaction between individuals materializes with a greater presence of non-verbal cues, body movement, and eye contact (Mehrabian, cited. in Rourke et al., 2001), groups prefer to meet face-to-face. According to the study results, the group preferred meeting face-toface because of the groups' preference for a higher degree of interaction. However, due to budget and geographical constraints, the group communicated via teleconferencing and web-conferencing, because of their inability to meet in face-to-face sessions.

In sum, there is a scarcity of research in this domain and healthcare groups prefer to collaborate using web-conferencing when they are unable to meet face-to-face because of financial costs and time constraints. Future studies should examine the impacts of conferencing technologies on academic detailing interventions with physicians; where instead of meeting face-to-face with clinicians to educate them on drug prescribing, they would do that virtually using web-conferencing. This would provide insight into the impacts of conferencing technologies on knowledge dissemination within an academic detailing environment.

\section{References}

1. Berg, B. (1989). Qualitative research methodology (3rd ed.). Boston: Allyn \& Bacon.

2. Canadian Institutes of Health Research [CIHR]. (2002). Knowledge translation overview. Ottawa: Retrieved December 15, 2004, from http://www.cihrirsc.gc.ca/about_cihr/organization/knowledge_translation/resources loverview_e.shtml.

3. Champion/Opinion. (2003). Leader/Knowledge Broker. Retrieved, from http://www.nursing.ualberta.ca/estabrooks/kusp/DatabaseOverview.htm.

4. Cramton, C. (2002) Finding common ground in dispersed collaboration. Organizational Dynamics, 30(4), 356-367.

5. Cramton, C. (2001). The mutual knowledge problem and its consequences for dispersed collaboration. Organization Science, 12(3), 346-371. 
6. Knowledge Utilization Studies in Practice [KUSP]. (2003). Overview of Champion/Opinion Leader/Knowledge Broker. Retrieved March 15, 2003, from http://www.nursing.ualberta.ca/estabrooks/kusp/DatabaseOverview.htm.

7. DeSanctis, G., \& Poole, M. (1994). Capturing the complexity in advanced technology use: Adaptive structuration theory. Organization Science, 5(2), 121-147.

8. Kondracki, N., Wellman, N., \& Amundson, D. (2002). Content analysis: review of methods and their applications in nutrition education. Journal of Nutrition Education and Behavior, 34(4), 224-230.

9. Lau, F., \& Hayward, R. (2000). Building a virtual network in a community health research training program. Journal of the American Medical Informatics Association, 7, 361-377.

10. O'Sullivan, E., \& Rassel, G.R. (1999). Research methods for public administrators (3rd ed.). New York: Addison Wesley Longman, Inc.

11. O'Brien, M.A., Rogers, S., Jamtvedt, G., Oxman, A.D., Odgaard-Jensen, J., Kristoffersen, D.T., Forsetlund, L., Bainbridge, D., Freemantle, N., Davis, D.A., Haynes, R.B., \& Harvey, E.L. (2007). Educational outreach visits: effects on professional practice and health care outcomes. Cochrane Database Syst Rev. 4:CD000409.

12. Quintana, Y., Nambayan, A., Ribeiro, R., Bowers, L., Shuler, A., \& O'Brien, R. (2003). Cure4Kids - building online learning and collaboration networks. AMIA Annu Symp Proc, 978.

13. Rourke, L., Anderson, T., Garrison, D.R., \& Archer, W. (2001). Assessing Social Presence in Asynchronous, Text-Based Computer Conferencing. Journal of Distance Education, 14(3), 51-70.

14. Soumerai, S. B., \& Avorn, J. (1990). Principles of educational outreach ('academic detailing') to improve clinical decision making. JAMA,263(4), 549-556.

15. Valaitis, R., Akhtar-Danesh, N., Eva, K., Levinson, A., \& Wainman, B. (2007). Pragmatists, positive communicators and shy enthusiasts: Three viewpoints about web conferencing in health sciences education. Journal of Medical Internet Research, 9(5), e-39.

16. Yin, R.K. (2003). Case study research. Thousand Oaks, CA: Sage. 